\title{
Elasticity in Macrophage-Synthesized Biocrystals
}

\author{
Elizabeth M. Horstman ${ }^{+}$Rahul K. Keswani ${ }^{+}$Benjamin A. Frey, Phillip M. Rzeczycki, \\ Vernon LaLone, Jeffery A. Bertke, Paul J. A. Kenis,* and Gus R. Rosania*
}

\begin{abstract}
Supramolecular crystalline assembly constitutes a rational approach to bioengineer intracellular structures. Here, biocrystals of clofazimine (CFZ) that form in vivo within macrophages were measured to have marked curvature. Isolated crystals, however, showed reduced curvature suggesting that intracellular forces bend these drug crystals. Consistent with the ability of biocrystals to elastically deform, the inherent crystal structure of the principal molecular component of the biocrystals - the hydrochloride salt of $\mathrm{CFZ}(\mathrm{CFZ}-\mathrm{HCl})$ - has a corrugated packing along the (001) face and weak dispersive bonding in multiple directions. These characteristics were previously found to be linked to the elasticity of other organic crystals. Internal stress in bent $\mathrm{CFZ}-\mathrm{HCl}$ led to photoelastic effects on the azimuthal orientation of polarized light transmittance. We propose that elastic, intracellular crystals can serve as templates to construct functional microdevices with different applications.
\end{abstract}

Abnormal morphology of crystals has been a subject of significant study over the last century. ${ }^{[1-6]}$ Particularly, crystalline growth and distribution within living organisms such as cells, tissues and clinically in humans has allowed an understanding of how solid-state crystal chemistry can modulate biological and biophysical environments. ${ }^{[7-12]}$ Specifically, the interaction of crystalline matter with cells such as macrophages within clinical microenvironments has garnered much attention. ${ }^{[13-15]}$ Macrophages (M $\varphi s$ ) are critical self-nonself recognizing immune cells capable of maintaining mammalian homeostasis and resolving inflammatory conditions responsible for many diseases. Concurrently, M $\varphi$ s are cellular

[*] E. M. Horstman, ${ }^{[+]}$Dr. P. J. A. Kenis

Department of Chemical and Biomolecular Engineering

University of Illinois, Urbana-Champaign

600 South Mathews Street, Urbana, IL 61801 (USA)

E-mail: kenis@illinois.edu

Dr. R. K. Keswani, ${ }^{[+]}$P. M. Rzeczycki, V. LaLone, Dr. G. R. Rosania Department of Pharmaceutical Sciences, College of Pharmacy

University of Michigan, Ann Arbor

428 Church Street, Ann Arbor, MI 48109 (USA)

E-mail: grosania@umich.edu

B. A. Frey

Morgan State University

1700 E Cold Spring Ln, Baltimore, MD 21251 (USA)

Dr. J. A. Bertke

School of Chemical Sciences

University of Illinois, Urbana-Champaign

505 South Mathews Street, Urbana, IL 61801 (USA)

$\left.{ }^{+}\right]$These authors contributed equally to this work.

(4) Supporting information and the ORCID identification number(s) for

(D) the author(s) of this article can be found under http://dx.doi.org/10. 1002/anie.201611195. "vacuum cleaners" eliminating foreign matter, typically referred to as xenobiotics. Such elimination may also be preceded by massive bioaccumulation, self-assembly and intracellular crystallization of sequestered foreign molecular agents such as clofazimine (CFZ), a red-pigmented, antiinflammatory, antimycobacterial, FDA-approved drug molecule. $^{[16,17]}$ Specifically, CFZ accumulates within M $\varphi$ s upon prolonged oral dosage in humans ${ }^{[18-21]}$ and rodent models ${ }^{[13,22-24]}$ (protocol in the Supporting Information (SI)) to form biocrystals containing clofazimine hydrochloride (CFZ-HCl) crystalline domains. ${ }^{[25]}$

In order to characterize the crystal structure and physical morphology of biocrystals, peritoneal and alveolar M $\varphi$ s from an 8-week CFZ-fed mouse were harvested. Through brightfield microscopy, several cell-associated dark red crystals were identified, some of which were surprisingly curved (Figure 1a). Moreover, after 10 minutes, the crystals turned over likely due to mechanical forces exerted by its parent $\mathrm{M} \varphi$ (Figure 1a). Upon closer inspection, we observed that another biocrystal in close proximity to the curved biocrystal also had a minor curvature that straightened out when the curved biocrystal flipped (Figure $1 \mathrm{a}$ ). In light of this empirical evidence, we further characterized the biocrystals for their curvature $(\kappa)$ within or isolated from M $\varphi$ s (Figure 1 b and S1 in the SI). The curvature per unit length $(\kappa / L)$ of biocrystals was significantly higher when present in peritoneal M $\varphi$ s (4.8fold change in mean, $p<0.005)$ or alveolar Mps (3.6-fold change in mean, $p<0.005)$ compared to when isolated from $\mathrm{M} \varphi \mathrm{s}$. A greater number of biocrystals were found to be inherently straight or having zero curvature when isolated (26) than when present in peritoneal M $\varphi s$ (10) or alveolar M $p s$ (18) (Figure 1b, S1). Such a reduction in curvature of isolated biocrystals are indicative of forces acting on the crystals curving them when present inside $\mathrm{M} \varphi \mathrm{s}$ and secondly of inherent elasticity of the biocrystals.

Mechanical properties of crystals (moduli, plasticity, elasticity) are known to be dependent on the atoms, ions or molecules forming the crystals and the interactions between these particles (molecular packing and intramolecular bonding). ${ }^{[2-30]}$ Further, recent work has explored the relationship between elasticity in organic crystals and their molecular and structural properties. Ghosh and Reddy reported an elastic and bendable organic cocrystal solvate formed from caffeine, 4-chloro-3-nitrobenzoic acid and methanol with weak and dispersive $\mathrm{C}-\mathrm{H} \cdots \pi$ interactions in three nearly perpendicular directions, suggesting that elastic deformation is due to isotropic molecular packing. ${ }^{[28,29]}$ Subsequently, Ghosh et al. studied $n$-benzylideneanilines and elucidated design rules for elastic organic crystals. ${ }^{[26-28]}$ Accordingly, elastic organic crystals should have 1) multi-directional weak dispersive bonds that can be easily broken to dissipate energy as the 


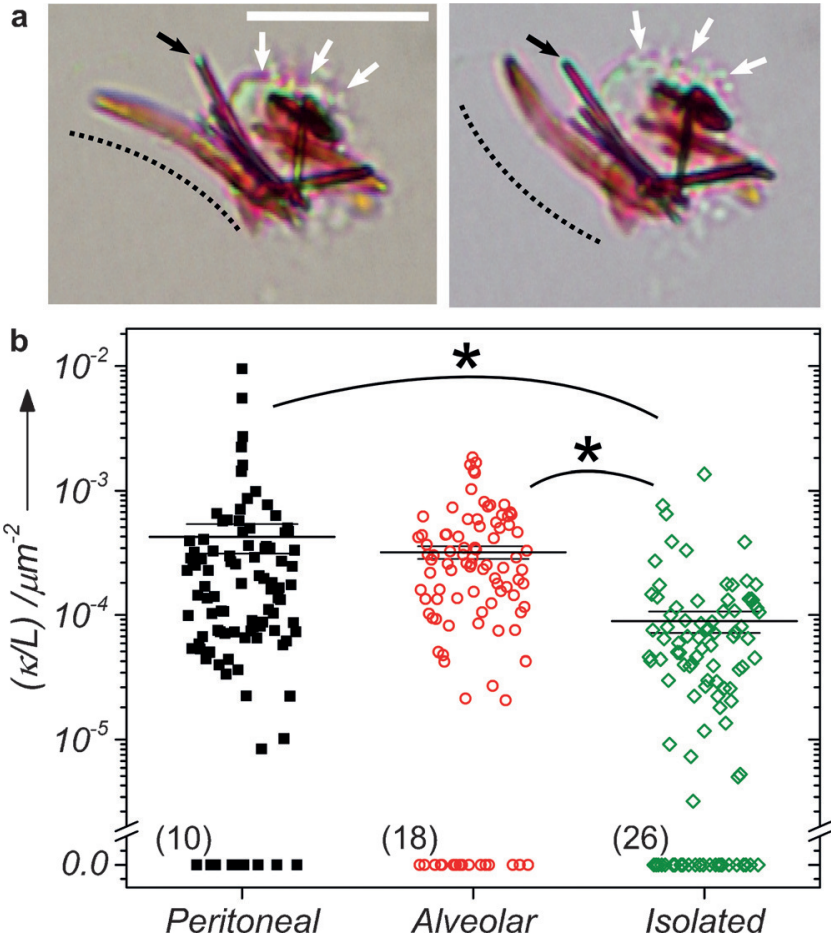

Figure 1. a) Peritoneal macrophages from a CFZ-fed mouse with the curvature of the curved biocrystal marked with dotted lines (white arrows show cell membrane, black arrows mark the crystal in physical contact with the first curved crystal). Scale bar: $10 \mu \mathrm{m}$. b) Curvature per unit arc length $(\kappa / L)$ of biocrystals in peritoneal $M \varphi s$ or alveolar Mys or when isolated from splenic Mps. $(n=100$ biocrystals, *: $p<0.005$. The wider and thick horizontal line indicates the mean of the distribution whereas the other smaller two lines indicate the mean \pm SE. Number in brackets indicates the number of biocrystals measured to have zero curvature.

crystal is bending and that can be easily formed when no force is applied and 2) corrugated packing along a crystallographic face to prevent long range dislocation within the crystal. ${ }^{[26-28]}$ In these studies, when crystals did not align with these design rules, they did not demonstrate elastic behavior. As such, we explored if elasticity and curvature of biocrystals could be explained via these design parameters.

As the primary component of the biocrystals is $\mathrm{CFZ}-\mathrm{HCl}$, we hypothesized that the inherent crystal structure of CFZ$\mathrm{HCl}$ plays a role in the flexibility (being able to adopt nonlinear morphologies) and elasticity (being able to return to linear conformations upon removal of force) of the biocrystals. A synthetic crystallization route was developed to grow CFZ-HCl crystals with similar crystal structure to the biocrystals (protocol in SI). The synthetic CFZ-HCl crystals form dark red rectangular plates (Figure 2a) that closely resemble the crystal habit and color of the biocrystals (Figure 2b) as observed with brightfield microscopy. The CFZ-HCl crystals can grow to be much larger than the biocrystals allowing for optimal structural characterization. The powder XRD (Figure 2c) suggests a strong agreement in the observed peaks of $\mathrm{CFZ}-\mathrm{HCl}$ with those from the biocrystals as previously reported. ${ }^{[25]}$ Both samples show strong preferential orientation of the crystals along the (001) face, consistent with face indexing of single crystals (Fig-

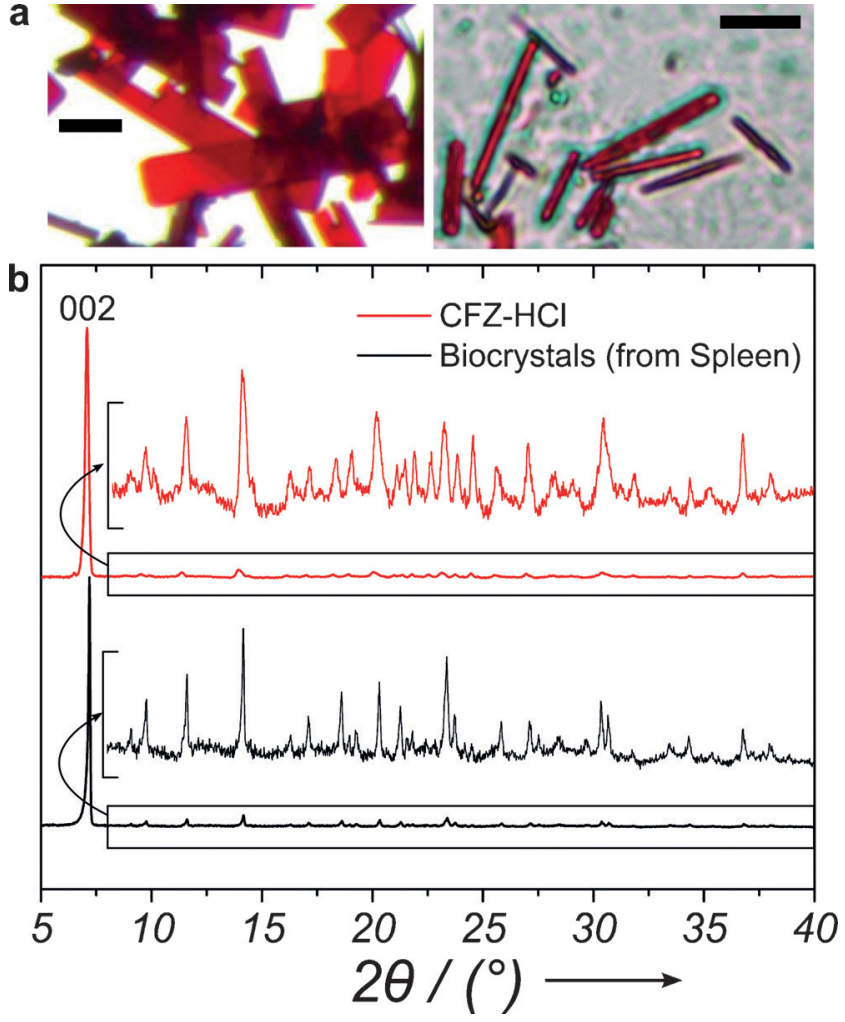

Figure 2. Optical micrograph of a) synthesized CFZ-HCl crystals, scale bar: $50 \mu \mathrm{m}$ and b) harvested biocrystals from a 8-week CFZ-fed mouse spleen, scale bar: $10 \mu \mathrm{m}$. c) Powder X-ray diffraction data from biocrystals and $\mathrm{CFZ}-\mathrm{HCl}$ crystals. Both samples display preferential orientation favoring the $(001)$ face. The diffraction data from $2 \theta=$ $8-40^{\circ}$ is zoomed in for convenient comparisons.

ure S2). The most intense peak on the p-XRD spectra is the (002) peak due to a systematic absence at $(00 l)$ when $l=2 n$ for Pbca space groups. Thus, the synthesized CFZ-HCl crystals are a suitable model for characterizing the mechanical behavior of the biocrystals.

Single-crystal XRD was also performed to determine the crystal structure of CFZ-HCl. The CFZ- $\mathrm{HCl}$ crystals grow in an orthorhombic $\mathrm{Pbca}$ space group with unit cell parameters; $a=10.266 \AA, b=19.828 \AA$, and $c=24.156 \AA, \alpha=\beta=\gamma=90^{\circ}$ and $Z=8$ (CCDC number: 1497722). One CFZ and one $\mathrm{HCl}$ molecule make up the asymmetric unit (Figure $3 \mathrm{a}$ ). $\mathrm{Cl} 3$ (the $\mathrm{Cl}$ associated with the $\mathrm{HCl}$ ) associates with the $\mathrm{CFZ}$ through two $\mathrm{N}-\mathrm{H} \cdots \mathrm{Cl}$ hydrogen bond interactions between $\mathrm{N} 3-$ $\mathrm{H} \cdots \mathrm{Cl} 3$ and $\mathrm{N} 4-\mathrm{H} \cdots \mathrm{Cl} 3(D, d, \theta: 3.172 \AA, 2.376 \AA$, 166.45 , and $\left.3.104 \AA, 2.243 \AA, 174.51^{\circ}\right)$. The data set was solved to $99.8 \%$ completeness. Water was incorporated into the crystal structure at an occupancy of 0.13 , and there was some disorder in the isopropyl group. The presence of water likely caused a change in the orientation of the isopropyl group, thereby contributing to the observed disorder (Figure S3). A summary of the unit cell parameters and diffraction data can be found in Table S1.

A key design feature of the $\mathrm{CFZ}-\mathrm{HCl}$ crystal structure is the corrugated packing along the (001) face (Figure $3 b, c$ ). One CFZ-HCl molecule makes up one step of the zig-zag and together the steps make up one corrugated sheet. The steps of 

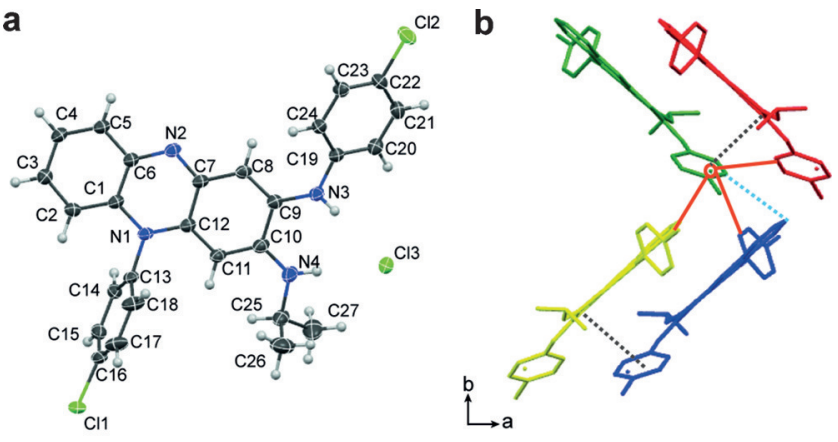

C

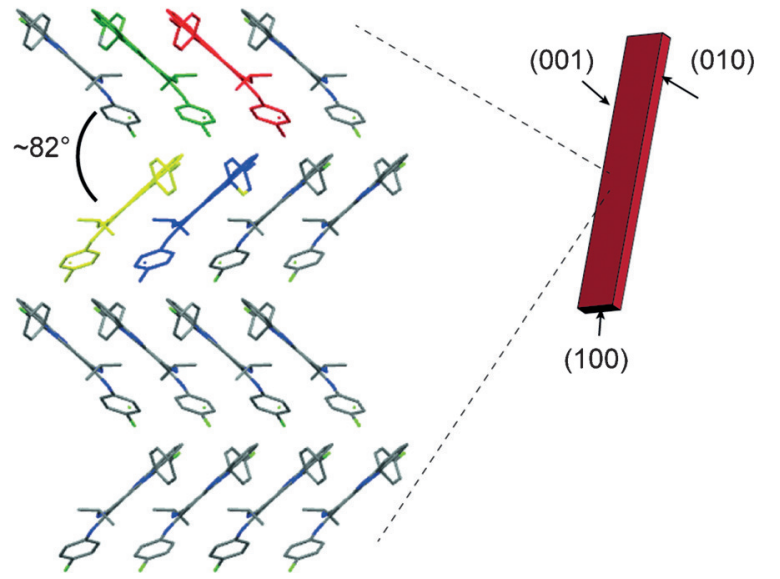

Figure 3. a) Asymmetric unit of CFZ-HCl displayed as an ellipsoid plot with $50 \%$ probability. The atomic positions for all non-hydrogen atoms are labeled. b) One section of the corrugated packing with solid orange lines indicating $\mathrm{C}-\mathrm{H} \cdots \mathrm{Cl}$ interactions, $\mathrm{C}-\mathrm{H} \cdots \pi$ indicated with a light blue dotted line and $\pi \cdots \pi$ interactions indicated by light grey dotted lines (unit cell looking along the $c$-axis). The molecules are displayed as capped sticks with the hydrogen atoms removed for better visualization of the crystals packing. c) Crystallographic projections of packing along the (001) face showing the corrugated packing. The projection is made up of $2 \times 2 \times 0.5(a \times b \times c)$ unit cells.

the zig-zag are held together with weak $\mathrm{C}-\mathrm{H} \cdots \pi$ and $\mathrm{C}-\mathrm{H} \cdots \mathrm{Cl}$ interactions, highlighted by light blue dotted lines and orange solid lines, respectively (Figure $3 \mathrm{~b}$ ). $\mathrm{Cl} 3$ is situated in the crease of the zig-zags and serves an anchor by coordinating with 3 additional molecules strengthening interactions within the crease and coordinating with adjacent sheets. Bond distances and angles are as follows: $\mathrm{C} 14-\mathrm{H} \cdots \mathrm{Cl} 3,3.503 \AA$, $2.724 \AA, 139.72^{\circ}, \mathrm{C} 24-\mathrm{H} \cdots \mathrm{Cl} 3,3.457 \AA, 2.757 \AA, 131.19^{\circ}$, and $\mathrm{C} 4-\mathrm{H} \cdots \mathrm{Cl} 3,3.625 \AA, 2.900 \AA$, $133.9^{\circ}$. These interactions are in 3 nearly perpendicular directions. Weak dispersive bonding between the sheets of the zig-zags from $\pi \cdots \pi$ interactions between the aromatic rings is also present (grey dotted lines,

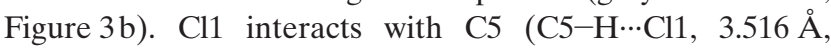
$\left.2.937 \AA, 120.48^{\circ}\right)$ to create a ribbon of $\mathrm{CFZ}$ molecules down the $b$-axis (Figure $\mathrm{S} 4$ ). When viewed along the $c$-axis this interaction is located in the crease and it stabilizes interactions between the sheets of the zig zag. As such, the weak multi-directional interactions are located at critical parts of the zig-zag, particularly associated with $\mathrm{Cl} 3$ and support the previously proposed design rules for crystal elasticity. ${ }^{[26]}$ The angle between the corrugated steps is $82^{\circ}$. All intermolecular interactions are listed in Table S2. Face indexing of the crystal during XRD showed that the (001) face is the largest surface which corresponds to the previous $\mathrm{p}-\mathrm{XRD}$ data. The projection of the (001) face is shown in Figure $3 \mathrm{c}$ to better show the corrugated packing. Projections of all the crystallographic faces can be found in Figure S5.

Interestingly, while harvesting $\mathrm{CFZ}-\mathrm{HCl}$ crystals for single crystals XRD, the crystals would noticeably bend when force was applied to pick up the crystals. As soon as the force was removed the crystals would promptly return to their original linear morphologies. Subsequently, video microscopy of bending crystals with tweezers was performed to further demonstrate their observed elastic response (Movie 1). Crystallization experiments yielded a very wide size distribution of crystals out of which crystals between $500 \mu \mathrm{m}$ and $2 \mathrm{~mm}$ long and ca. $20 \mu \mathrm{m}$ thick were chosen. The CFZ-HCl crystals typically lie with the (001) face normal to the substrate (Figure $4 \mathrm{a}$ ). When a slight force was applied, the crystal rotated $90^{\circ}$ about the $a$-axis (Figure $4 \mathrm{~b}$ ). When a force was applied to the (001) face, the crystal bent (Figure 4c,d) and assumed its original shape upon removal of the force (Figure $4 \mathrm{e}$ ). The crystal arched again when a second force was applied, Figure $4 \mathrm{f}$. As such, the crystals can be bent many times without noticeable deformation to the crystal. In one experiment, a crystal was bent 8 times without noticeable deformation (Movie 2). However, upon applying a larger force, the crystal snapped and the crystalline fragments adopted the original straight configuration (Movies 1 and 2).

To confirm the elastic deformation of $\mathrm{CFZ}-\mathrm{HCl}$, polarization microscopy was performed as a way of measuring the

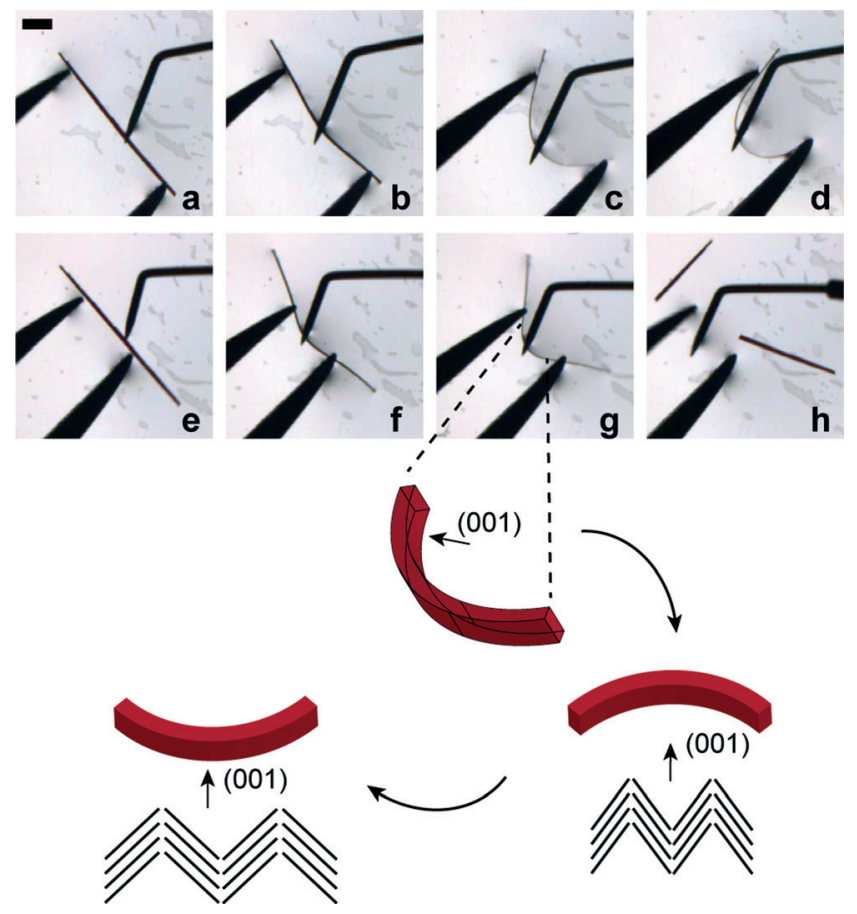

Figure 4. a-d) A CFZ-HCl crystal was bent by applying a force with a crystallization probe while the crystal was held stationary against a pair of tweezers. e) The force was removed and the crystal quickly regained the original position before $f$ ) another force was applied to the crystal. Scale bar is $100 \mu \mathrm{m}$. The bottom schematic shows how the angle of corrugation would look like on the convex and concave edges of the crystal during bending. 
photoelastic phenomena that is commonly observed with application of stress and development of strain. ${ }^{[31]}$ In this particular study, linear dichroism could be measured using hardware configured on an epifluorescence-brightfield microscope $^{[32,33]}$ (see the SI for protocol). Crystals were imaged under polarized light following which the anisotropy in transmittance and importantly, the azimuth (the polarization orientation that results in maximum transmittance) were computed (Figure 5). ${ }^{[34]}$ Crystals were first identified as regions with low transmittance (Figure $5 \mathrm{a}, \mathrm{c}$ ). Given the structural packing of the $\mathrm{CFZ}-\mathrm{HCl}$ lattice and absence of polycrystalline elements in the biocrystals, the azimuth distribution was expectedly isotropic across the crystal plane when unstressed and straight (Figure 5b). In contrast, the azimuth was anisotropic in the elastically deformed region of the crystal wherein two approximately orthogonal azimuthal orientations were measured (Figure $5 \mathrm{~d}$ ). In the concave region, the azimuth was perpendicular to the long axis of the crystal whereas in the convex region, the azimuth was retained along the long-axis of the crystal as observed in the straight crystals. Such anisotropy is clearly indicative of a stress network resulting in the development of an elastic strain through the crystal. ${ }^{[35]}$ As such, given the extent of cross-interactions within the crystal lattice as described before (Figure 3, S2-S4), the development of an elastic stress-strain network through an expectedly isotropic crystal structure was accomplished and verified through photoelastic azimuth measurements.

Further, no differences in vibrational spectra (Raman) were evident before and after bending at the point of

a
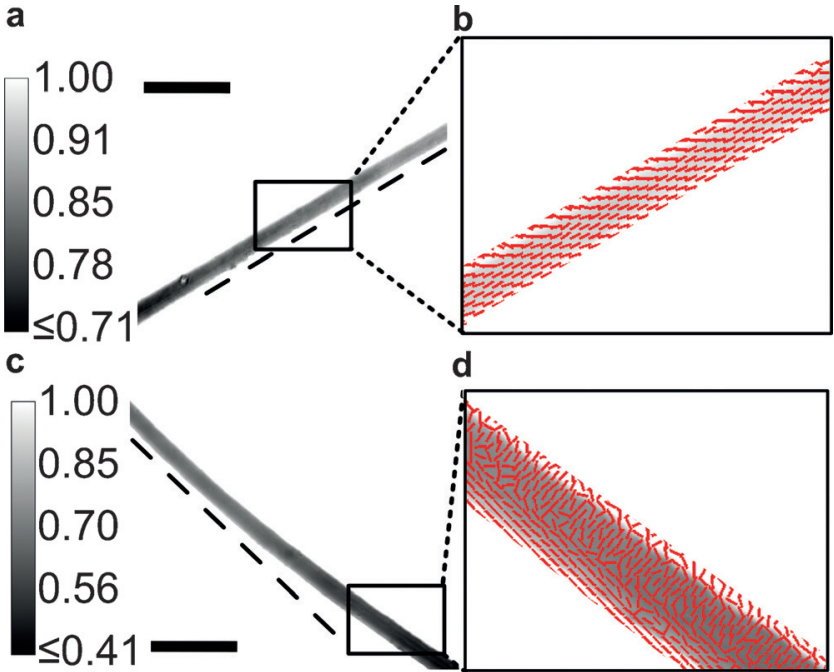

Figure 5. a) Computed transmittance image of a a,b) straight vs. $c, d)$ bent crystal. Calibration bar in $(a, c)$ shows the range of transmittance. Dark regions (with low transmittance) follow the crystal with the dotted tangential line from one edge of the crystal confirming the curvature in the crystal. Azimuth: polarization orientation of maximum transmittance overlaid on the transmittance image indicating unidirectional axes in the b) straight crystal while in the d) bent crystal, there is a bidirectional axes depending on the direction of compressional (inward bending edge leads to an orthogonal axes) or tensile stress (outward bending edge leading to a longitudinal axis). Scale bar in $(\mathrm{a}, \mathrm{b})$ is $100 \mu \mathrm{m}$. maximum curvature in these crystals (Figure S6). Naturally curved crystals also showed no differences in molecular vibrations compared to the reference $\mathrm{CFZ}-\mathrm{HCl}$ crystals. Finally, the melting point for $\mathrm{CFZ}-\mathrm{HCl}$ was measured to be $275^{\circ} \mathrm{C}$ (Figure S7), which is $53^{\circ} \mathrm{C}$ degrees higher than $\mathrm{CFZ}$ while other salts of $\mathrm{CFZ}$ also have melting points $<246^{\circ} \mathrm{C}^{[36]}$ As indicated by their melting points, the salts of CFZ are more stable than CFZ. The stabilization of CFZ as CFZ-HCl within mammalian cells therefore favors the formation of a highly stable crystalline polymorph and as reported here has appropriate structural packing features, reinforced by the additional $\mathrm{Cl}$ from $\mathrm{HCl}(\mathrm{Cl} 3$ in Figure $3 \mathrm{a})$ that allow it to adapt to intracellular mechanical stressors.

In summary, we show that the inherent structural packing features of CFZ-HCl within biocrystals indeed contribute to their elasticity and naturally adjustable curvature. As such, formation of biocrystals mediated by M $\varphi s$ leads to the development of elastic and curved crystalline elements with structural packing features that showcase the classical design rules designated for organic crystal flexibility. ${ }^{[26-29]}$ The presence of CFZ biocrystals in the $\mathrm{M} \varphi$ endows them with potent anti-inflammatory characteristics ${ }^{[37,38]}$ and specific fluorescent ${ }^{[39]}$ and photoacoustic ${ }^{[40,41]}$ signatures for cellular optical tracking. Such dual therapeutic and diagnostic (theranostic) applications make biocrystals a potent cellular device that could be harnessed for biomedical applications. М $\varphi$ mediated crystallization is an important example of how these cells self-assemble a crystal with features that allow for easier adaptability to the mechanical environment of the cell, thereby allowing a massive drug loading within the cell. Importantly, the additional $\mathrm{Cl}$ (in $\mathrm{HCl}$ ) is most likely reinforced within CFZ biocrystals through the presence of multiple chloride channels in macrophages and their highly regulated role in cellular physiology ${ }^{[42-44]}$ Here, we show that the salt chloride also plays an integral role in the structural stability and intracellular mechanical adaptation of biocrystals via elasticity.

Furthermore, mechanical flexibility of exogenous elements within cells could be a critical design parameter toward engineering organic intracellular constructs to endow cells with unnatural yet stable and beneficial features for therapeutic applications. Finally, viscoelasticity, pressures and connected mechano-transductive elements are connected to inflammatory cell phenotypes. ${ }^{[45-49]}$ As such, the mechanochemical characterization of intracellular properties could be important for cell-based mechano-biological applications leading to a new class of pharmaceutics: mechano-pharmaceutics.

\section{Acknowledgements}

We thank the National Science Foundation (NSF) for a Graduate Research Fellowship to E.M.H. (DGE1144245), National Institute of General Medical Sciences (NIGMS) for funding support to G.R.R. (R01GM078200), University of Michigan of Office of Research MCubed Program funding to G.R.R., Michigan Institute for Clinical \& Health Research Pilot Seed funding to G.R.R. and R.K.K. 
(NIH: UL1TR000433) and the Interdisciplinary REU (Research Experiences for Undergraduates) Program housed in the College of Pharmacy, University of Michigan for funding B.A.F. through the NSF-Division of Biological Infrastructure (NSF-DBI) (DBI-1263079). We acknowledge the support of Dr. Gislaine Kuminek, Dr. Nair RodriguezHornedo, Dr. Gi Sang Yoon and Sudha Sud (Department of Pharmaceutical Sciences, College of Pharmacy, University of Michigan) for help with DSC and animal experiments, Dr. Danielle Gray, George L. Clark (X-ray Facility and 3M Materials Laboratory, University of Illinois) for discussion about crystallographic data, and Lucas C. Gonzalez (Department of Chemical and Biomolecular Engineering, University of Illinois) for help with crystallization experiments.

\section{Conflict of interest}

Gus Rosania is a consultant for Bristol-Myers Squibb. The other authors have no conflict of interest.

Keywords: biocrystals · clofazimine $\cdot$ crystal engineering elasticity $\cdot$ X-ray diffraction

How to cite: Angew. Chem. Int. Ed. 2017, 56, 1815-1819 Angew. Chem. 2017, 129, 1841-1845

[1] L. J. Spencer, Mineral. Mag. 1921, 19, 263-274.

[2] M. K. Panda, S. Ghosh, N. Yasuda, T. Moriwaki, G. D. Mukherjee, C. M. Reddy, P. Naumov, Nat. Chem. 2015, 7, 65-72.

[3] C. M. Reddy, R. C. Gundakaram, S. Basavoju, M. T. Kirchner, K. A. Padmanabhan, G. R. Desiraju, Chem. Commun. 2005, 3945-3947.

[4] H. Koshima, R. Matsuo, M. Matsudomi, Y. Uemura, M. Shiro, Cryst. Growth Des. 2013, 13, 4330-4337.

[5] K. Godwod, A. T. Nagy, Z. Rek, Phys. Status Solidi A 1976, 34 , 705.

[6] F. Terao, M. Morimoto, M. Irie, Angew. Chem. Int. Ed. 2012, 51, 901-904; Angew. Chem. 2012, 124, 925-928.

[7] J. Baumgartner, G. Morin, N. Menguy, T. Perez Gonzalez, M. Widdrat, J. Cosmidis, D. Faivre, Proc. Natl. Acad. Sci. USA 2013 110, 14883-14888.

[8] M. I. Siponen, P. Legrand, M. Widdrat, S. R. Jones, W.-J. Zhang, M. C. Y. Chang, D. Faivre, P. Arnoux, D. Pignol, Nature 2013 $502,681-684$.

[9] Y. Wang, A. Lomakin, T. Hideshima, J. P. Laubach, O. Ogun, P. G. Richardson, N. C. Munshi, K. C. Anderson, G. B. Benedek, Proc. Natl. Acad. Sci. USA 2012, 109, 13359-13361.

[10] P. F. Weller, E. J. Goetzl, K. F. Austen, Proc. Natl. Acad. Sci. USA 1980, 77, 7440-7443.

[11] S. R. Mulay, H.-J. Anders, N. Engl. J. Med. 2016, 374, 2465-2476.

[12] D. J. Sullivan, Jr., I. Y. Gluzman, D. E. Goldbery, Science 1996 271, 219-222.

[13] R. T. Aplin, A. C. McDougall, Experientia 1975, 31, 468-469.

[14] P. Vicari, V. M. Sthel, N. Engl. J. Med. 2015, 373, e27.

[15] F. Martinon, V. Pétrilli, A. Mayor, A. Tardivel, J. Tschopp, Nature 2006, 440, 237-241.

[16] M. C. Cholo, H. C. Steel, P. B. Fourie, W. A. Germishuizen, R Anderson, J. Antimicrob. Chemother. 2012, 67, 290-298.

[17] V. C. Barry, J. G. Belton, M. L. Conalty, J. M. Denneny, D. W. Edward, J. F. O’Sullivan, D. Twomey, F. Winder, Nature 1957, 179, 1013-1015.

[18] L. Levy, Am. J. Trop. Med. Hyg. 1974, 23, 1097-1109.
[19] P. Belaube, J. Devaux, M. Pizzi, R. Boutboul, Y. Privat, Int. J. Lepr. Other Mycobact. Dis. 1983, 51, 328-330.

[20] D. K. Banerjee, G. A. Ellard, P. T. Gammon, M. F. R. Waters, Am. J. Trop. Med. Hyg. 1974, 23, 1110-1115.

[21] A. C. McDougall, W. R. Horsfall, J. E. Hede, A. J. Chaplin, Br. J. Dermatol. 1980, 102, 227-230.

[22] J. Baik, K. A. Stringer, G. Mane, G. R. Rosania, Antimicrob. Agents Chemother. 2013, 57, 1218-1230.

[23] J. Baik, G. R. Rosania, PLoS One 2012, 7, e47494.

[24] M. L. Conalty, R. D. Jackson, Br. J. Exp. Pathol. 1962, 43, 650654.

[25] R. K. Keswani, J. Baik, L. Yeomans, C. Hitzman, A. Johnson, A. Pawate, P. J. A. Kenis, N. Rodríguez-Hornedo, K. A. Stringer, G. R. Rosania, Mol. Pharm. 2015, 12, 2528-2536.

[26] S. Ghosh, M. K. Mishra, S. B. Kadambi, U. Ramamurty, G. R. Desiraju, Angew. Chem. Int. Ed. 2015, 54, 2674-2678; Angew. Chem. 2015, 127, 2712-2716.

[27] S. Ghosh, M. K. Mishra, S. Ganguly, G. R. Desiraju, J. Am. Chem. Soc. 2015, 137, 9912-9921.

[28] S. Ghosh, C. M. Reddy, Angew. Chem. Int. Ed. 2012, 51, 1031910323; Angew. Chem. 2012, 124, $10465-10469$.

[29] C.-T. Chen, S. Ghosh, C. M. Reddy, M. J. Buehler, Phys. Chem. Chem. Phys. 2014, 16, 13165-13171.

[30] S. Hayashi, T. Koizumi, Angew. Chem. Int. Ed. 2016, 55, $2701-$ 2704; Angew. Chem. 2016, 128, 2751-2754.

[31] H. Mueller, Phys. Rev. 1935, 47, 947-957.

[32] K. A. Min, W. G. Rajeswaran, R. Oldenbourg, G. Harris, R. K. Keswani, M. Chiang, P. Rzeczycki, A. Talattof, M. Hafeezma, R. Horobin, et al., Adv. Sci. 2015, 2, 1500025

[33] M. Koike-Tani, T. Tani, S. B. Mehta, A. Verma, R. Oldenbourg, Mol. Reprod. Dev. 2015, 82, 548-562.

[34] S. B. Mehta, M. Shribak, R. Oldenbourg, J. Opt. 2013, 15, 094007.

[35] A. Curtis, L. Sokolikova-Csaderova, G. Aitchison, Biophys. J. 2007, 92, 2255-2261.

[36] G. Bolla, A. Nangia, Cryst. Growth Des. 2012, 12, 6250-6259.

[37] G. S. Yoon, S. Sud, R. K. Keswani, T. J. Standiford, K. A. Stringer, G. R. Rosania, Mol. Pharm. 2015, 12, 2517 - 2527.

[38] G. S. Yoon, R. K. Keswani, S. Sud, P. Rzeczycki, M. Murashov, T. Koehn, T. J. Standiford, K. A. Stringer, G. R. Rosania, Antimicrob. Agents Chemother. 2016, 60, 3470-3479.

[39] R. K. Keswani, G. S. Yoon, S. Sud, K. A. Stringer, G. R. Rosania, Cytom. Part A 2015, 87, 855-867.

[40] C. Tian, R. K. Keswani, G. Gandikota, G. R. Rosania, X. Wang, Proc. SPIE 2016, 9708, 97084L-1.

[41] R. K. Keswani, C. Tian, T. Peryea, G. Girish, X. Wang, G. R. Rosania, Sci. Rep. 2016, 6, 23528.

[42] T. J. Jentsch, J. Physiol. 2007, 578, 633-640.

[43] A. R. Graves, P. K. Curran, C. L. Smith, J. A. Mindell, Nature 2008, 453, 788-792

[44] L. Jiang, K. Salao, H. Li, J. M. Rybicka, R. M. Yates, X. W. Luo, X. X. Shi, T. Kuffner, V. W.-W. Tsai, Y. Husaini, et al., J. Cell Sci. 2012, 125, 5479-5488.

[45] N. R. Patel, M. Bole, C. Chen, C. C. Hardin, A. T. Kho, J. Mih, L. Deng, J. Butler, D. Tschumperlin, J. J. Fredberg, et al., PLoS One 2012, 7, e41024.

[46] K. M. Adlerz, H. Aranda-Espinoza, H. N. Hayenga, Eur. Biophys. J. 2016, 45, 301-309.

[47] J. Pugin, I. Dunn, P. Jolliet, D. Tassaux, J. L. Magnenat, L. P. Nicod, J. C. Chevrolet, Am. J. Physiol. 1998, 275, L1040-L1050.

[48] H. Shiratsuchi, M. D. Basson, Am. J. Physiol. Cell Physiol. 2004, 286, C1358-C1366.

[49] H. Y. Shin, D. M. Frechette, N. Rohner, X. Zhang, D. A. Puleo, L. M. Bjursten, J. Tissue Eng. Regener. Med. 2013, 2, 408-417.

Manuscript received: November 15, 2016

Final Article published: January 12, 2017 